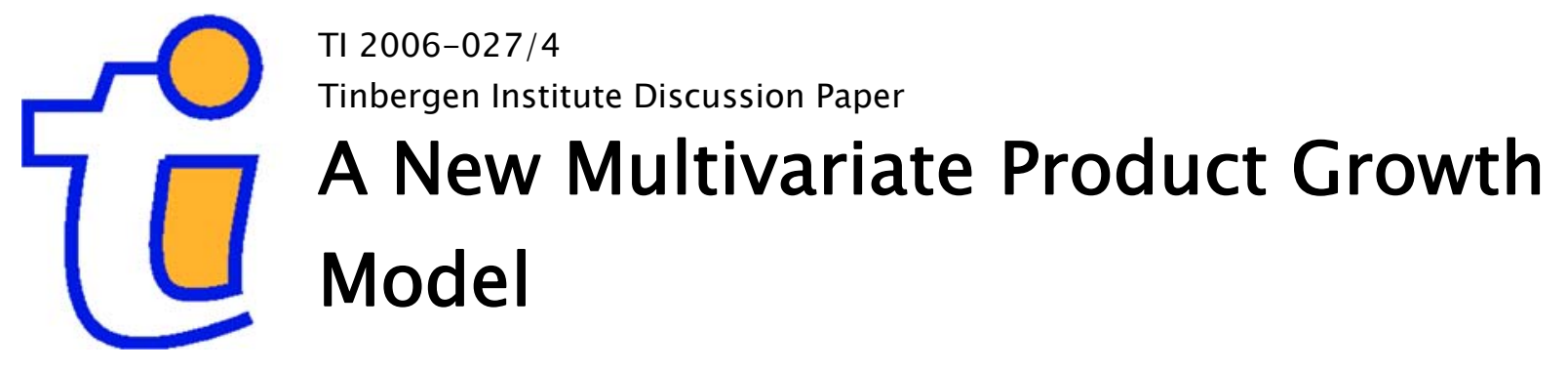

H. Peter Boswijk 1,3

Dennis Fok ${ }^{2,3}$

Philip Hans Franses2,3

' Universiteit van Amsterdam;

2 Erasmus Universiteit Rotterdam;

3 Tinbergen Institute. 


\title{
A new multivariate product growth model
}

\author{
H. Peter Boswijk \\ Department of Quantitative Economics \\ Universiteit van Amsterdam
}

\author{
Dennis Fok* \\ Econometric Institute \\ Erasmus University Rotterdam \\ Philip Hans Franses \\ Econometric Institute \\ Erasmus University Rotterdam
}

March 15, 2006

*Corresponding author. Address: Econometric Institute, Erasmus School of Economics and Business Economics, Erasmus University Rotterdam, P.O. Box 1738, NL-3000 DR Rotterdam, The Netherlands, email: dfok@few.eur.nl, phone +31 10 4081333, fax +31 104089162 


\title{
A new multivariate product growth model
}

\begin{abstract}
To examine cross-country diffusion of new products, marketing researchers have to rely on a multivariate product growth model. We put forward such a model, and show that it is a natural extension of the original Bass (1969) model. We contrast our model with currently in use multivariate models and we show that inference is much easier and interpretation is straightforward. In fact, parameter estimation can be done using standard commercially available software. We illustrate the benefits of our model relative to other models in simulation experiments. An application to a three-country CD sales series shows the merits of our model in practice.
\end{abstract}

Keywords: Diffusion, international marketing, econometric models 


\section{Introduction}

The Bass (1969) model is often used in marketing research to describe and to forecast the empirical adoption curve of new products and technological innovations. It basically has three unknown parameters, which characterize the important features of a variety of S-shaped curves that are typical for the diffusion process of new products. As the number of parameters is small, the Bass model is usually considered for a single annual series, where the time span concerns 10 to 20 years.

Ever since its inception, the Bass model witnessed a large amount of modifications and extensions in various directions. These model versions aim to capture more dynamics, additional explanatory variables, sequences of generations, and also, more than one diffusion variable. In this paper we address this last case, that is, the extension of the basic Bass model to allow for two or more diffusion series, which somehow may be correlated with each other. Hence, we propose a model for multivariate new product growth. In this paper we present the model in terms of a cross-country diffusion analysis, however the model can also be applied to other multivariate diffusion processes, such as the (joint) diffusion of multiple products in the same country.

There are various multivariate versions of the Bass model around, see Putsis et al. (1997) and Kumar and Krishnan (2002) for example. We review these models in Section 2, where we also show that these models suffer from the critique summarized in Bass et al. (1994). This basic critique says that if the diffusion of adoptions may depend on an explanatory variable, which itself is strongly correlated with time, then the basic Bass model without any such variables would fit about equally well. Hence, if country A's diffusion would be correlated with that in country B, then adding country B's diffusion to the model for country A would not contribute much to the fit. In fact, a single country Bass model would fit equally well. In econometric language, the explanatory variables for country B's diffusion process are too collinear with the explanatory variables for the diffusion process in country A, that proper parameter estimates are difficult or impossible to obtain.

In the same Section 2 we put forward a new multivariate product growth model, that does not have the above-mentioned problems. Our model extends in a natural way the empirical Bass-type model as it is proposed in Boswijk and Franses (2005), and hence it builds naturally on the original Bass model. The basic idea in Boswijk and Franses (2005) is that a univariate diffusion series follows an S-shaped path, from which short- 
lived deviations are possible. The speed of adjustment towards this path is given by an adjustment parameter, which in the final empirical model implies the inclusion of an additional lagged adoption variable. Additionally, Boswijk and Franses (2005) propose to modify the original Bass model with heteroskedastic error terms. Such an extension is also rather straightforward for our new multivariate model, as we will demonstrate in the empirical illustration.

Our multivariate extension of the Boswijk and Franses (2005) model and hence Bass model allows for cross-equation adjustments to country-specific paths. In other words, deviations from the path in country A have an effect on the deviations from the underlying adoption process in country B. Next, we allow for contemporaneous correlation. After discretizing, our model is a system of equations with parameter constraints. This system can easily be estimated by (nonlinear) Generalized Least Squares. We provide the relevant Eviews code in Appendix 1.

In Section 3, we report on the outcomes of a limited simulation experiment in which we compare the various multivariate Bass models. For each model we generate diffusion processes and calculate the root mean squared error for all the multivariate diffusion models. We show that diffusion paths generated by the earlier proposed multivariate diffusion models can indeed be replicated almost perfectly by simple univariate Bass models. The model of Boswijk and Franses (2005), and by extension our model, allows for the case of a stochastic diffusion process. In a second experiment we consider the influence of the relative size of the stochastic component on the performance of the different models.

In Section 4, we illustrate the usefulness of the various models on data for CD adoption in the US, Canada and Japan. We give the data in Appendix 2. We show that our model yields insights in the relation between different countries. For example, we show that crosscountry diffusion may not be symmetric. Finally, in Section 5 we summarize limitations and ideas for further research. 


\section{Multivariate product growth models}

In this section we discuss four variants of multivariate product growth models, where we consider the case of two countries to save notation. Extensions to more than two countries follow straightforwardly. We first consider a simple extension of the basic Bass model to the case of two countries. Then we consider two models that have been proposed recently, that is the model of Putsis et al. (1997), which we assign the acronym PBKS, and the model of Kumar and Krishnan (2002), which we will label the KK model. Finally, we introduce our model, for which we use the acronym BFF. At the start, we will use the commonly used notation in continuous time, while towards the end we will discuss the discretized versions of the models.

We denote $n_{i}(t)$ as the continuous time increments of the product growth process of country $i$ and $N_{i}(t)$ as the level of adoption, that is, $n_{i}(t)=d N_{i}(t) / d t$. The basic Bass model reads as

$$
\frac{n_{i}(t)}{1-N_{i}(t)}=p_{i}+q_{i} N_{i}(t)
$$

where it is assumed here that 1 marks the maturity level of the adoption process. The parameter $p_{i}$ is called the innovation parameter, and $q_{i}$ is the imitation parameter. For sake of notation, to become useful below, we rewrite this equation as

$$
n_{i}(t)=\left(1-N_{i}(t)\right)\left(p_{i}+q_{i} N_{i}(t)\right)=n_{i}^{*}(t) .
$$

Note that the left hand side of the equation now contains the variable of interest that one wants to explain, that is, the growth, while the right hand side only depends on the current installed base at time $t$. Throughout this paper we will denote the implied growth rates according to the Bass model by $n_{i}^{*}(t)$. Modifying this expression to allow for a maturity level $m_{i}$ is well known to be straightforward, but for ease of notation we abstain from this for the moment.

\subsection{Currently available models}

A natural extension of this single-equation Bass model to cover countries 1 and 2, could be written as

$$
\left(\begin{array}{l}
n_{1}(t) \\
n_{2}(t)
\end{array}\right)=\left(\begin{array}{cc}
1-N_{1}(t) & 0 \\
0 & 1-N_{2}(t)
\end{array}\right)\left[\left(\begin{array}{l}
p_{1} \\
p_{2}
\end{array}\right)+\left(\begin{array}{cc}
q_{1} & 0 \\
0 & q_{2}
\end{array}\right)\left(\begin{array}{l}
N_{1}(t) \\
N_{2}(t)
\end{array}\right)\right]=\left(\begin{array}{l}
n_{1}^{*}(t) \\
n_{2}^{*}(t)
\end{array}\right)
$$

This diagonal multivariate Bass $[\mathrm{DMB}]$ model assumes that each of the two countries has its own adoption process and that these two processes are everywhere independent. 
We take this model as a benchmark model when we study in the simulation experiments whether the same issues as noted in Bass et al. (1994) extend to the multivariate case.

It might however be possible that the diffusion processes in the countries 1 and 2 interact somehow. A first proposal of a multivariate model is done in Putsis et al. (1997), which, in our notation can be written as

$$
\left(\begin{array}{l}
n_{1}(t) \\
n_{2}(t)
\end{array}\right)=\left(\begin{array}{cc}
1-N_{1}(t) & 0 \\
0 & 1-N_{2}(t)
\end{array}\right)\left[\left(\begin{array}{l}
p_{1} \\
p_{2}
\end{array}\right)+\left(\begin{array}{ll}
q_{11} & q_{12} \\
q_{21} & q_{22}
\end{array}\right)\left(\begin{array}{l}
N_{1}(t) \\
N_{2}(t)
\end{array}\right)\right]
$$

Clearly, the key difference between the diagonal multivariate Bass model and this one is in the $2 \times 2$ matrix with the imitation parameters. This can be seen even more clearly by writing the PBKS model as

$$
\left(\begin{array}{l}
n_{1}(t) \\
n_{2}(t)
\end{array}\right)=\left(\begin{array}{l}
n_{1}^{*}(t) \\
n_{2}^{*}(t)
\end{array}\right)+\left(\begin{array}{cc}
1-N_{1}(t) & 0 \\
0 & 1-N_{2}(t)
\end{array}\right)\left(\begin{array}{cc}
0 & q_{12} \\
q_{21} & 0
\end{array}\right)\left(\begin{array}{l}
N_{1}(t) \\
N_{2}(t)
\end{array}\right)
$$

From a theoretical point of view, this extension makes much sense. Indeed, it can be the case that the level of the diffusion in a neighboring country exercises an effect on the own country diffusion. On the other hand, note that this model only allows for a positive effect of one country on another. The cross-country influence is modeled through the number of contacts in one country with another country. Furthermore, as $N_{i}(t)$ measures the number of cumulative adoptions, $n_{i}(t)$ must be positive, and this also leads to the restriction that $q_{i j}>0$.

From a practical point of view, the PBKS model in (5) might not be easy to handle. The main reason is that it suffers from exactly the same collinearity problems as the generalized Bass model does, as noted convincingly in Bass et al. (1994). Indeed, it is most likely that both $N_{1}(t)$ and $N_{2}(t)$ have an S-shaped pattern that is strongly correlated with time, and with each other. Hence adding say $N_{2}(t)$ to an equation for $N_{1}(t)$ makes it difficult to estimate the parameters $q_{11}$ and $q_{12}$. As an extreme example, consider the case where the diffusion patterns in two countries follow the same S-shape, but are in fact unrelated. In this case the correlation between $N_{1}(t)$ and $N_{2}(t)$ will be almost perfect. It is now impossible to estimate the $q_{i j}$ parameters. Furthermore, in case the diffusion curves are different and interrelated, the additional explanatory power of the cumulative number of adoptors $\left(N_{2}(t)\right)$ to explain the diffusion in country 1 may be very limited. In our simulation experiments below, we will demonstrate this phenomenon.

Ignoring these possible multicollinearity problems, estimation of the PBKS model parameters is rather straightforward. After discretizing the continuous time model in (5) 
one can apply least squares to obtain estimates of the model parameters, see Section 2.3 for more details.

A second multivariate model that has been proposed recently in the marketing literature is given in Kumar and Krishnan (2002). In their notation it reads as

$$
\begin{aligned}
& \frac{n_{1}(t)}{1-N_{1}(t)}=\left(p_{1}+q_{1} N_{1}(t)\right)\left(1+b_{21} n_{2}(t)\right) \\
& \frac{n_{2}(t)}{1-N_{2}(t)}=\left(p_{2}+q_{2} N_{2}(t)\right)\left(1+b_{12} n_{1}(t)\right)
\end{aligned}
$$

which in fact seems very close to a multivariate version of the generalized Bass model. To make it comparable with the two models above, and with our model still to come, we rewrite this model first as

$$
\left(\begin{array}{l}
n_{1}(t) \\
n_{2}(t)
\end{array}\right)=\left(\begin{array}{cc}
n_{1}^{*}(t) & 0 \\
0 & n_{2}^{*}(t)
\end{array}\right)\left[\left(\begin{array}{l}
1 \\
1
\end{array}\right)+\left(\begin{array}{cc}
0 & b_{21} \\
b_{12} & 0
\end{array}\right)\left(\begin{array}{l}
n_{1}(t) \\
n_{2}(t)
\end{array}\right)\right]
$$

where $n_{i}^{*}(t)$ is defined in (2). We can rewrite this equation as

$$
\left[\mathbf{I}-\left(\begin{array}{cc}
n_{1}^{*}(t) & 0 \\
0 & n_{2}^{*}(t)
\end{array}\right)\left(\begin{array}{cc}
0 & b_{21} \\
b_{12} & 0
\end{array}\right)\right]\left(\begin{array}{l}
n_{1}(t) \\
n_{2}(t)
\end{array}\right)=\left(\begin{array}{l}
n_{1}^{*}(t) \\
n_{2}^{*}(t)
\end{array}\right),
$$

where $\mathbf{I}$ denotes a $2 \times 2$ identity matrix. Solving this equation for the $2 \times 1$ vector $n(t)$ containing the country-specific diffusion series gives

$$
\left(\begin{array}{l}
n_{1}(t) \\
n_{2}(t)
\end{array}\right)=\left[\mathbf{I}-\left(\begin{array}{cc}
n_{1}^{*}(t) & 0 \\
0 & n_{2}^{*}(t)
\end{array}\right)\left(\begin{array}{cc}
0 & b_{21} \\
b_{12} & 0
\end{array}\right)\right]^{-1}\left(\begin{array}{l}
n_{1}^{*}(t) \\
n_{2}^{*}(t)
\end{array}\right),
$$

provided that the inverse exists. It is clear that the KK model nests the diagonal multivariate Bass model as setting $b_{12}=0$ and $b_{21}=0$ in the KK model yields the other model. An empirical comparison of these two models can simply be done using likelihood ratio tests, say. Comparing the KK model with the PBKS model is not straightforward, however.

The expression in (9) shows that this multivariate model is highly non-linear in variables as well as in parameters, as it contains the inverse of a matrix with elements that contain parameters and variables. This may make the theoretical interpretation of this model not easy. Also, at first sight one may expect that this should make parameter estimation not very easy, and this is confirmed in Kumar and Krishnan (2002). Indeed, these authors describe a rather complicated estimation routine, which also seems to have problems to deliver standard errors (as these are not reported in their tables). 
For parameter estimation in the simulation experiments below, we consider an alternative procedure to the one proposed in Kumar and Krishnan (2002). Instead of estimating the "structural" model (6), we consider the "reduced form" (9). We apply the common discretization techniques and estimate the model parameters using least squares, see also Section 2.3.

\subsection{A new multivariate model}

To overcome the potential problems raised above concerning currently available multivariate models, we intend to propose a model that is easy to interpret and of which the parameters are easy to estimate. The main idea is to extend the error correction type expression in equation (6) of Boswijk and Franses (2005) to the multivariate setting. Boswijk and Franses (2005) consider the basic univariate Bass model and address the issue of the stochastic nature of the diffusion process. Generally, in practice, the original Bass equation in continuous time is discretized and than simply an error term is added, which is assumed to have mean zero and common variance. Boswijk and Franses (2005) argue that this approach of adding an error term does not match with the original notions behind the Bass theory. In fact, it makes more sense to assume that theoretical diffusion follows a deterministic S-shaped path, around which the actually observed diffusion process fluctuates where these fluctuations are caused by random events, individual-specific characteristics, or by marketing-mix effects, say. These fluctuations are however such that there always is a tendency to return to the theoretical underlying deterministic S-shape. This implies that there is, say, a target or attractor-like diffusion process, around which the actual diffusion fluctuates around while preserving a tendency to return to that target level.

In the notation of the current paper, the key equation in Boswijk and Franses (2005) for a single country is

$$
d n_{i}(t)=\alpha_{i}\left(n_{i}^{*}(t)-n_{i}(t)\right) d t+\sigma_{i} n_{i}(t)_{i}^{\gamma} d W_{i}(t)
$$

where $W_{i}(t)$ is a standard Brownian motion. The actual diffusion series $n_{i}(t)$ wanders around the target diffusion $n_{i}^{*}(t)$, where deviations from this target are caused by random shocks driven by $W_{i}(t)$. The size of the random shocks are proportional to $n_{i}(t)^{\gamma}$. Over time the $n_{i}(t)$ returns to the target path, the speed of this error correction is determined by $\alpha_{i}$. The target path in this model is exactly the path according to the Bass diffusion 
model (2). Note that the model is now specified in terms of changes in the growth rate, that is, $d n_{i}(t)$.

The BF model explicitly allows for random events to influence the diffusion. Diffusion curves that correspond with this model do not necessarily show a perfect S-shape. This is an important feature of this model, as in practice one also does not always encounter perfect curves.

A multivariate version of this error correction type model, to be labeled as BFF, is given by the two equations

$$
\left(\begin{array}{l}
d n_{1}(t) \\
d n_{2}(t)
\end{array}\right)=\left(\begin{array}{ll}
\alpha_{11} & \alpha_{12} \\
\alpha_{21} & \alpha_{22}
\end{array}\right)\left(\begin{array}{l}
n_{1}^{*}(t)-n_{1}(t) \\
n_{2}^{*}(t)-n_{2}(t)
\end{array}\right) d t+\left(\begin{array}{l}
\sigma_{1} n_{1}(t)^{\gamma} d W_{1}(t) \\
\sigma_{2} n_{2}(t)^{\gamma} d W_{2}(t)
\end{array}\right)
$$

where $W_{1}(t)$ and $W_{2}(t)$ are possibly correlated Brownian motions. The off-diagonal elements of the matrix $\alpha$, that is, $\alpha_{12}$ and $\alpha_{21}$, have a straightforward interpretation. They can be interpreted as the effect that deviations in one country have on the deviations from the underlying diffusion path in another country. For example, suppose that $\alpha_{12}<0$ and $n_{2}(t)<n_{2}^{*}(t)$, that is, the actual diffusion in country 2 is below its target path. The product $\alpha_{12}\left(n_{2}^{*}(t)-n_{2}(t)\right)$ is then also negative, meaning that the diffusion in country 1 will slow down. Hence, deviations from the target paths in each of the countries also have an effect on the changes in the diffusion in the other country. As in the univariate case, these deviations can be due to marketing-mix effects.

When we restrict $\alpha_{12}=\alpha_{21}=0$ we end up with two stacked BF models, where the random shocks may be correlated. One could call this model a diagonal BFF model. Below we refer to this model as the BF model.

After discretization, the parameters in the BFF model can simply be estimated using Generalized (nonlinear) Least Squares, and estimated standard errors can be obtained along the usual lines. Also, the additional variables in the BFF model do not have patterns that come close to trends or sigmoid shape trends, and hence, the BFF model does not have the problems noted in Bass et al. (1994). We present more on estimation below. Furthermore, as a courtesy to the reader, we give the Eviews code for a bivariate BFF model in Appendix 1.

In sum, the currently available multivariate models seem to suffer from potential estimation problems, while the BFF model does not. Additionally, the BFF model has easy to interpret parameters from a marketing point of view. To illustrate the estimation issues mentioned, we turn to a report of simulation experiments, and we postpone an empirical 
illustration to Section 4. Before doing so, we say a few words about discretization and estimation.

\subsection{Discretization and estimation}

All models discussed above are in continuous time. In practice we need to fit these models to, say, annual data, that is, data measured at discrete intervals. The diffusion models therefore have to be transformed to discrete time. The equations to use for parameter estimation follow naturally from these transformations. In general we index the observation moments using a subscript $k$, where observation $k$ corresponds to time $t=k \delta, k=0, \ldots, T / \delta$, where $\delta$ denotes the length of the time interval between two observations. In most practical cases $\delta=1$. The observation moments are then $t=0$, $t=1, \ldots, t=T$. To keep the presentation as general as possible, we use the subscript $k$ to denote the observation moments. For example, $N_{i, k}$ denotes the cumulative adoption in country $i$ at observation moment $t=k \delta$.

Discretizing the deterministic models (DMB, PBKS, and KK) is rather straightforward. For $t=k \delta$ one can simply replace the $n_{i}(t)$ on the left-hand side of the equations by $X_{i, k} / \delta$, where $X_{i, k}=\Delta N_{i, k}=N_{i, k}-N_{i, k-1}$, and the $N_{i}(t)$ appearing on the right-hand side by $N_{i, k-1}$. For the discretization of the stochastic components in the BF and BFF model we replace the component $d n_{i}(t)$ on the left-hand side of the equation by $\Delta X_{i, k}$, for the right-hand side we replace $N_{i}(t)$ by $N_{i, k-1}, n_{i}(t)$ by $X_{i, k-1}$, and $d t$ by $\delta$, we refer to Boswijk and Franses (2005) for more details. The discretization of $d W_{i}(t)$ yields independent identically distributed random variables with variance $\delta$.

For parameter estimation in the DMB, PBKS and KK models one can straightforwardly minimize the sum of squared errors in the discretized models. Note that this estimation procedure for the KK model is far more straightforward than that proposed in Kumar and Krishnan (2002). For the BF and BFF models one can choose one of two options. The first option is to ignore differences in variance across series and possible correlations across error terms, and simply minimize the sum of squared errors over all observations. We opt for this procedure in the simulation experiments below. For the empirical section we take the stochastic component seriously and estimate the parameters by applying (nonlinear) GLS to the discretized version of the model. One can account for heteroskedasticity by dividing the equations for country $i$ through $X_{i, k-1}$. Note that this option corresponds to setting $\gamma=1$ in (11). 


\section{Simulation experiments}

In this section we illustrate the performance of the different multivariate models discussed above. The setup of the experiment is as follows. We select one of the models and generate data according to that model. Next, each of the 5 models is fitted to the generated data. Using the estimated parameters the models are used to generate forecasts of the growth figures. We measure the forecasting performance by the root mean squared error [RMSE] of the forecasts. Comparing the RMSE of the different models gives us insight into how much the models differ from each other in the implied diffusion curves. For example, when for data generated with a given model, the DMB yields roughly the same RMSE as the true model, one can conclude that the latter model does not add much to the DMB model. Possible explanations for findings such as this one are the problems first noted in Bass et al. (1994).

Except for the $\mathrm{BF}$ and the BFF model, the (multivariate) diffusion models do not have a stochastic component. That is, given the model parameters, the diffusion curve is fixed. Therefore we first consider the deterministic case, where for BF and BFF we set $\sigma_{i}=0$. Afterwards we consider what happens when the BF or the BFF models are used as DGP with $\sigma_{i} \neq 0$.

\section{Deterministic models}

For each data generating process $[\mathrm{DGP}]$, that is, for DMB, PBKS, KK, BF and BFF, we generate data for $T=20$ periods. To generate data we use the discretizations as discussed in Section 2.3 but now with $\delta=0.001$, that is, we very closely approximate continuous time. For model estimation and diffusion forecasting we of course only use the observations for $t=0,1,2, \ldots, T$. Next, each of the models is fitted to these data. For example, we generate data from (2), and fit models (2), (5), (9), and (11), and so on. We compute the root mean squared error of the sales figures $(N(t)-N(t-1)$, for $t=2, \ldots, T)$, and we average these over 1000 replications.

To generate data that look like the typical data obtained in practice, we have to set proper parameter values. The illustrations in the various studies that put forward the models under scrutiny provided a source of inspiration. For DMB model we draw values for the imitation and innovation parameters in country 1 and 2 from two uniform distributions, that is 


$$
\begin{aligned}
p_{i} & \sim \mathrm{U}[0.005,0.055], \\
q_{i} & \sim \mathrm{U}[0.15,0.55],
\end{aligned}
$$

where $i$ is 1 or 2 . For the PBKS model we interpret $q_{i}$ from (12) as the total external influence, we divide this influence over the within-country influence $\left(q_{i i}\right)$ and the acrosscountry influence $\left(q_{i j}\right)$ using

$$
\begin{aligned}
f_{i} & \sim \mathrm{U}[0,0.5], \\
q_{i i} & =\left(1-f_{i}\right) q_{i}, \\
q_{i j} & =f_{i} q_{i} .
\end{aligned}
$$

To make sure that the resultant bivariate series make sense we restrict the cross-country influence to be smaller than the within-country influence. Unreported graphs of the series substantiate this claim. Next, for the KK model we use the same way to generate $p_{i}$ and $q_{i}$ as in the DMB model, where we additionally draw the $b_{i j}$ parameters according to

$$
b_{i j} \sim \mathrm{U}[0,0.75]
$$

For the univariate BF model we additionally need the error correction parameters, and we draw these according to

$$
\alpha_{i} \sim \mathrm{U}[2,10]
$$

Finally, the BFF requires error correction parameters across countries, and these we generate according to

$$
\begin{aligned}
r_{i} & \sim \mathrm{U}[-0.9,0.9] \\
\alpha_{i j} & =r_{i} \alpha_{i i} .
\end{aligned}
$$

Again we restrict the size of cross-country effect to be smaller than the within-country effect. Note that the BFF model also allows for a negative correlation between countries, which is in contrast to the PBKS model. In sum, we have 5 sets of DGPs, and in the first round of experiments, we also fit 5 models for each DGP. To allow for a fair forecast comparison, we fit all models by minimizing the sum of the squared residuals.

The simulation results for the deterministic case are given in Table 2. The first panel gives the root mean squared errors when averaged over 1000 replications. Note that the DGPs in this case are deterministic, that is, conditional on the parameters the diffusion 
figures are fixed. In principle, the model of the DGP would fit the data perfectly. The reason that we do not find zero RMSE in Table 2 is that there are discretization errors. The data are generated in (almost) continuous time, while we estimate the model for discrete time.

The second panel gives these figures again, but now scaled towards each of the DGPs. Hence, the value 1 should appear when the same model is fitted to the data generated by that model. The first panel already indicates that differences can be quite large, and this can even be better seen from the second panel. For example, when the data are generated by an DMB model, then the root mean squared error of the PBKS model is 0.92 times that of the DMB model itself. And, when the data are generated by an BFF model, the RMSE of the KK model is 3.4 times as large that of the BFF model. Clearly, the results in Table 2 indicate that it is best to fit the BFF model when the data are generated by any of the other models. This improvement in fit is of course partly because the BFF model contains the most parameters. Note however that the BF model also outperforms the KK and PBKS models, while they have an equal number of parameters.

The differences in performance of the PBKS and KK models versus the DMB model are quite small, even when the data are generated using PBKS or KK. This is mainly due to the problems noted in Bass et al. (1994). The additional regressors in the PBKS and KK models do not add much in explanatory power. In fact the generated diffusion curves very closely resemble curves that can be fitted using the standard Bass model. The poor fit of the DMB model in case BF and BFF are the DGPs is due to the fact that the DMB lacks relevant variables.

\section{Nonzero error variance}

Our next set of simulations concerns the cases where the BF and BFF models are the DGP, where we now allow an error term to enter the model with a nonzero variance. Note that the main difference in the BF model versus the original Bass model lies in the fact that the BF model allows for stochastic variation in the diffusion. In the multivariate case it is exactly the stochastic variation that helps us to identify cross-country influences. Note that we impose zero contemporaneous correlation between the error terms.

In Table 3 we report similar results as we did in the second panel of Table 2, which can also be seen from the first row of each panel. The results in Table 3 are easy to interpret. When the DGP is BF, and the error variance is small, then the BFF model improves the fit. This is of course due to the fact that the BFF model contains more 
parameters. Qualitatively similar results appear when the BFF model is the DGP. A second important result from these experiments is that when the error variance becomes larger, the differences in fit become smaller. Indeed, it might be expected that more variation in the data leads to decreased performance for all models. Once the signal to noise ratio in the data becomes very low, the differences between the models disappear as there is only noise to fit.

In sum, the simulation results in this section clearly show that the new multivariate product growth model outperforms other models in terms of fit, even when it is not the data generating process.

\section{Cross country effects of CD diffusions}

We now turn to an illustration of the new multivariate model for new product growth, also to demonstrate its relevance for actually observed data. We consider annual time series running from 1983 to 1996 concerning CD sales in the US, Canada and Japan. The data are given in Appendix 2, and the graphs of these three series appear in Figure 1. When we compute the correlation between these series, we get 0.996 for the US with Canada, 0.953 for the US with Japan, and 0.929 for Canada with Japan. These high values already suggest that the PBKS and KK models would run into estimation problems, so these models are not considered here.

We fit the BFF model while allowing for heteroskedasticity. We follow the suggestions in Boswijk and Franses (2005) that dividing the left-hand side and the right-hand side of the equations trough $X_{j, k-1}$ can take care of it, hence we set $\gamma=1$ in (11) and we do not follow a formal empirical testing strategy for $\gamma$. Hence, the model that we actually estimate is

$$
\begin{aligned}
&\left(\begin{array}{c}
\frac{\Delta X_{1, k}}{X_{1, k-1}} \\
\frac{\Delta X_{2, k}}{X_{2, k-1}} \\
\frac{\Delta X_{3, k}}{X_{3, k-1}}
\end{array}\right)=\left(\begin{array}{ccc}
\frac{1}{X_{1, k-1}} & & \\
& \frac{1}{X_{2, k-1}} & \\
& \frac{1}{X_{3, k-1}}
\end{array}\right)\left(\begin{array}{ccc}
\alpha_{11} & \alpha_{12} & \alpha_{13} \\
\alpha_{21} & \alpha_{22} & \alpha_{23} \\
\alpha_{31} & \alpha_{32} & \alpha_{33}
\end{array}\right) \times \\
&\left(\begin{array}{l}
\left(m_{1}-N_{1, k-1}\right)\left(p_{1}+q_{1} N_{1, k-1} / m_{1}\right) \\
\left(m_{2}-N_{2, k-1}\right)\left(p_{2}+q_{2} N_{2, k-1} / m_{2}\right) \\
\left(m_{3}-N_{3, k-1}\right)\left(p_{3}+q_{3} N_{3, k-1} / m_{3}\right)
\end{array}\right)+\left(\begin{array}{l}
\varepsilon_{1, k} \\
\varepsilon_{2, k} \\
\varepsilon_{3, k}
\end{array}\right),
\end{aligned}
$$

where $X_{i, k}=N_{i, k}-N_{i, k-1}$.

The estimation results for the three-country BFF model appear in Figure 2, where 
we present the actual Eviews output. Normally, we would not like to see such tables in academic papers, but we do include it here to show that the BFF model with heteroskedasticity can actually be estimated using a simple Eviews program. In Table 4, we provide the relevant parameter estimates in a more common format. The estimation results in Figure 2 as well as in Table 4 show that there are effects running from the US to Canada and Japan, as the estimated parameters $\alpha_{21}$ and $\alpha_{31}$ are significant at the 5 per cent level. Both $\alpha_{21}$ as $\alpha_{31}$ are negative, this implies that when the development of the diffusion in the US falls behind the target path the same will happen in Canada and in Japan. The same holds for the reverse case, if the diffusion in the US goes faster than the target path, the diffusion in Canada and Japan will also speed up. Other cross-country effects are insignificant, see Table 4. The implied $p, q$ and $m$ parameters of the standard Bass model also seem reasonable, see the middle panel of Table 4.

In sum, we see that the new multivariate version of a Bass model can be fitted quite easily to a trivariate series, that it delivers interpretable and meaningful parameters, and that it has a high in-sample fit.

\section{Conclusion}

In this paper we have put forward a new multivariate product growth model, and we contrasted it with other such multivariate models using theoretical and simulation-based arguments. Given the outcomes of the simulations, and also of the empirical illustration, we are tempted to conclude that our new model outperforms its rivals on various dimensions. Further applications should substantiate our claim, and also out-of-sample forecasting contests should even further do so. Together with designing a specification strategy for the best way to incorporate heteroskedasticity, we leave these issues for further research. 


\section{Appendix 1: Eviews code for a bivariate BFF model with heteroskedasticity}

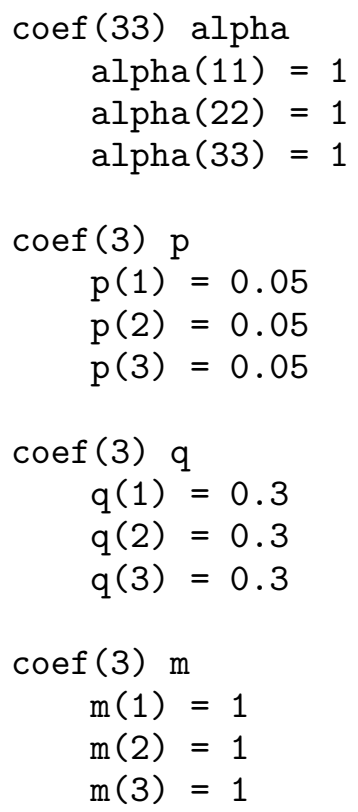

BFF.sur

Note: The series usa, can, and jpn are the cumulative diffusions for the USA, Canada, and Japan, respectively. 


\section{Appendix 2: CD diffusion in three countries}

Table 1: Cumulative CD sales in the USA, Canada and Japan.

\begin{tabular}{cccc}
\hline Year & USA & Canada & Japan \\
\hline 1983 & 0.001763 & 0.000000 & 0.011117 \\
1984 & 0.010578 & 0.007250 & 0.040811 \\
1985 & 0.042465 & 0.019174 & 0.134696 \\
1986 & 0.101068 & 0.048887 & 0.284236 \\
1987 & 0.163569 & 0.119618 & 0.391979 \\
1988 & 0.222702 & 0.173152 & 0.497629 \\
1989 & 0.301163 & 0.230174 & 0.653189 \\
1990 & 0.386953 & 0.336152 & 0.747037 \\
1991 & 0.477370 & 0.451939 & 0.895274 \\
1992 & 0.524994 & 0.527244 & 0.914487 \\
1993 & 0.592513 & 0.578241 & 0.929264 \\
1994 & 0.655934 & 0.663102 & 0.934292 \\
1995 & 0.725858 & 0.747712 & 0.935567 \\
1996 & 0.773400 & 0.787030 & 0.946600 \\
\hline
\end{tabular}



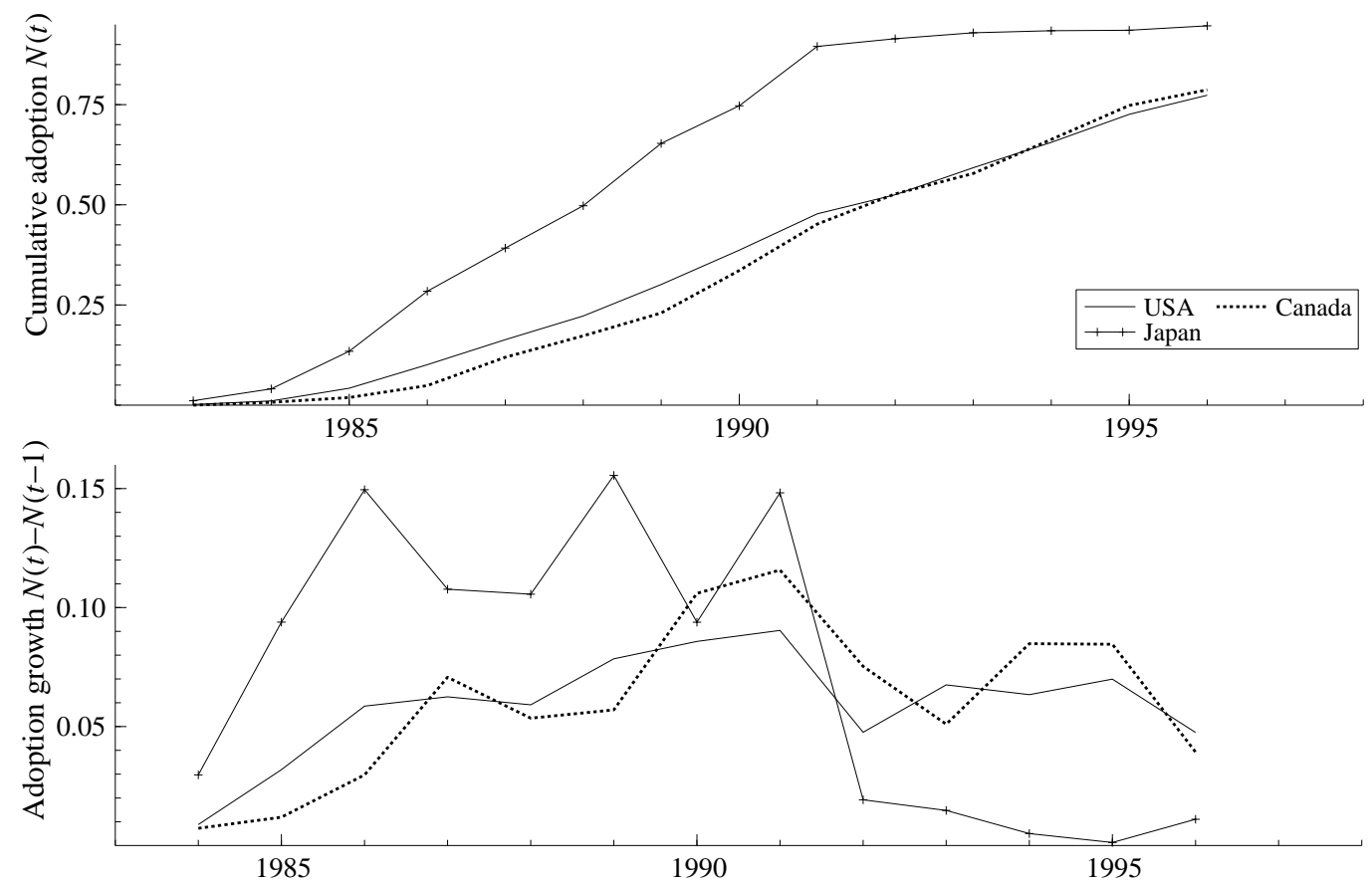

Figure 1: Graphical representation of the diffusion data 


\section{Other tables and figures}

\begin{tabular}{|c|c|c|c|c|}
\hline \multicolumn{5}{|c|}{$\begin{array}{l}\text { System: BFF } \\
\text { Estimation Method: Seemingly Unrelated Regression } \\
\text { Date: 03/15/06 Time: 16:01 } \\
\text { Sample: } 19851996 \\
\text { Included observations: } 12 \\
\text { Total system (balanced) observations } 36 \\
\text { Iterate coefficients after one-step weighting matrix } \\
\text { Convergence achieved after: } 1 \text { weight matrix, } 15 \text { total coef iterations }\end{array}$} \\
\hline & Coefficient & Std. Error & t-Statistic & Prob. \\
\hline ALPHA(11) & 0.156122 & 0.253043 & 0.616978 & 0.5450 \\
\hline$M(1)$ & 0.904784 & 0.123548 & 7.323354 & 0.0000 \\
\hline$P(1)$ & 0.036593 & 0.019512 & 1.875409 & 0.0771 \\
\hline$Q(1)$ & 0.300414 & 0.088667 & 3.388101 & 0.0033 \\
\hline ALPHA(12) & 0.326078 & 0.216728 & 1.504546 & 0.1498 \\
\hline$M(2)$ & 0.853710 & 0.070692 & 12.07645 & 0.0000 \\
\hline$P(2)$ & 0.038881 & 0.017231 & 2.256493 & 0.0367 \\
\hline$Q(2)$ & 0.391601 & 0.086228 & 4.541481 & 0.0003 \\
\hline ALPHA(13) & 0.134733 & 0.107192 & 1.256933 & 0.2248 \\
\hline$M(3)$ & 0.941106 & 0.011748 & 80.11025 & 0.0000 \\
\hline$P(3)$ & 0.093492 & 0.033463 & 2.793869 & 0.0120 \\
\hline $\mathrm{Q}(3)$ & 0.514120 & 0.101584 & 5.061024 & 0.0001 \\
\hline ALPHA(21) & -1.068347 & 0.370388 & -2.884401 & 0.0099 \\
\hline ALPHA(22) & 1.253604 & 0.267560 & 4.685321 & 0.0002 \\
\hline ALPHA(23) & -0.035655 & 0.160001 & -0.222842 & 0.8262 \\
\hline ALPHA(31) & -0.478949 & 0.215553 & -2.221960 & 0.0393 \\
\hline ALPHA(32) & 0.048026 & 0.128257 & 0.374452 & 0.7124 \\
\hline $\operatorname{ALPHA}(33)$ & 1.002103 & 0.356141 & 2.813779 & 0.0115 \\
\hline \multicolumn{5}{|c|}{ Determinant residual covariance $\quad 0.000123$} \\
\hline \multicolumn{5}{|c|}{$\begin{array}{l}\left.\text { Equation: (USA+USA }(-2)-2^{*} \mathrm{USA}(-1)\right) /(\mathrm{USA}(-1)-\mathrm{USA}(-2))=(\mathrm{ALPHA}(11) \\
{ }^{*}\left((\mathrm{M}(1)-\mathrm{USA}(-1))^{\star}\left(\mathrm{P}(1)+\mathrm{Q}(1)^{*} \mathrm{USA}(-1) / \mathrm{M}(1)\right)-(\mathrm{USA}(-1)-\mathrm{USA}(-2))\right) \\
+\mathrm{ALPHA}(12)^{*}\left((\mathrm{M}(2)-\mathrm{CAN}(-1))^{\star}\left(\mathrm{P}(2)+\mathrm{Q}(2)^{\star} \mathrm{CAN}(-1) / \mathrm{M}(2)\right)-(\mathrm{CAN}(\right. \\
-1)-\mathrm{CAN}(-2)))+\mathrm{ALPHA}(13)^{*}\left((\mathrm{M}(3)-\mathrm{JPN}(-1))^{\star}\left(\mathrm{P}(3)+\mathrm{Q}(3)^{\star} \mathrm{JPN}(-1)\right.\right. \\
\text { /M(3)) - (JPN }(-1)-\mathrm{JPN}(-2)))) /(\mathrm{USA}(-1)-\mathrm{USA}(-2)) \\
\text { Observations: } 12\end{array}$} \\
\hline R-squared & 0.952446 & \multirow{3}{*}{\multicolumn{2}{|c|}{$\begin{array}{l}\text { Mean dependent var } \\
\text { Sum squared resid }\end{array}$}} & \multirow{3}{*}{$\begin{array}{l}0.300680 \\
0.338017\end{array}$} \\
\hline S.D. dependent var & 0.803860 & & & \\
\hline Durbin-Watson stat & 3.127232 & & & \\
\hline \multicolumn{5}{|c|}{$\begin{array}{l}\text { Equation: }\left(\mathrm{CAN}+\mathrm{CAN}(-2)-2^{*} \mathrm{CAN}(-1)\right) /(\mathrm{CAN}(-1)-\mathrm{CAN}(-2))=( \\
\text { ALPHA }(21)^{\star}\left((\mathrm{M}(1)-\mathrm{USA}(-1))^{*}\left(\mathrm{P}(1)+\mathrm{Q}(1)^{*} \mathrm{USA}(-1) / \mathrm{M}(1)\right)-(\mathrm{USA}(-1)\right. \\
\text {-USA }(-2)))+\mathrm{ALPHA}(22)^{*}\left((\mathrm{M}(2)-\mathrm{CAN}(-1))^{\star}\left(\mathrm{P}(2)+\mathrm{Q}(2)^{*} \mathrm{CAN}(-1)\right.\right. \\
\text { /M(2)) - (CAN }(-1)-\mathrm{CAN}(-2)))+\mathrm{ALPHA}(23)^{\star}\left((\mathrm{M}(3)-\mathrm{JPN}(-1))^{\star}(\mathrm{P}(3)\right. \\
\left.\left.\left.\text { +Q(3) }(3)^{\star} \mathrm{JPN}(-1) / \mathrm{M}(3)\right)-(\mathrm{JPN}(-1)-\mathrm{JPN}(-2))\right)\right) /(\mathrm{CAN}(-1)-\mathrm{CAN}(-2)) \\
\text { Observations: } 12\end{array}$} \\
\hline R-squared & 0.880187 & \multirow{3}{*}{\multicolumn{2}{|c|}{$\begin{array}{l}\text { Mean dependent var } \\
\text { Sum squared resid }\end{array}$}} & \multirow{3}{*}{$\begin{array}{l}0.311958 \\
0.616074\end{array}$} \\
\hline S.D. dependent var & 0.683705 & & & \\
\hline Durbin-Watson stat & 2.716070 & & & \\
\hline \multicolumn{5}{|c|}{ 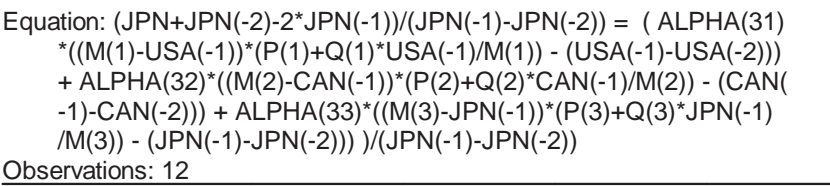 } \\
\hline R-squared & 0.974530 & \multirow{3}{*}{\multicolumn{2}{|c|}{$\begin{array}{l}\text { Mean dependent var } \\
\text { Sum squared resid }\end{array}$}} & 0.687833 \\
\hline S.D. dependent var & 2.344713 & & & 1.540288 \\
\hline Durbin-Watson stat & 2.142392 & & & \\
\hline
\end{tabular}

Figure 2: Estimation results (actual Eviews output) 
Table 2: Simulation results for the deterministic case $(\sigma=0)$, where DMB denotes the diagonal multivariate Bass model, PBKS is the model in Putsis et al. (1997), KK is the model of Kumar and Krishnan (2002), BF is the univariate model in Boswijk and Franses (2005) and BFF is the new multivariate model.

\begin{tabular}{lccccc}
\hline \multicolumn{5}{c}{ Estimated model } \\
DGP & DMB & PBKS & KK & BF & BFF \\
\hline \multicolumn{5}{c}{ Root mean squared errors } \\
DMB & 0.219 & 0.201 & 0.166 & 0.044 & 0.039 \\
PBKS & 0.223 & 0.190 & 0.155 & 0.082 & 0.074 \\
KK & 0.217 & 0.201 & 0.160 & 0.041 & 0.037 \\
BF & 0.131 & 0.125 & 0.099 & 0.032 & 0.028 \\
BFF & 0.146 & 0.140 & 0.118 & 0.053 & 0.035 \\
Root mean squared errors relative to DGP \\
DMB & 1 & 0.917 & 0.757 & 0.201 & 0.178 \\
PBKS & 1.173 & 1 & 0.818 & 0.431 & 0.388 \\
KK & 1.356 & 1.257 & 1 & 0.254 & 0.231 \\
BF & 4.088 & 3.899 & 3.095 & 1 & 0.872 \\
BFF & 4.162 & 3.990 & 3.376 & 1.527 & 1 \\
\hline
\end{tabular}


Table 3: Simulation results for the cases with nonzero variance, where DMB denotes the diagonal multivariate Bass model, PBKS is the model in Putsis et al. (1997), KK is the model of Kumar and Krishnan (2002), BF is the univariate model in Boswijk and Franses (2005) and BFF is the new multivariate model, and where the BF and BFF models are the DGP. The numbers give the RMSE relative to the RMSE when the data are fitted using the correct model.

\begin{tabular}{lccccc}
\hline$\sigma$ & DMB & PBKS & KK & BF & BFF \\
\hline \multicolumn{5}{c}{ DGP: BF } \\
0 & 4.088 & 3.899 & 3.095 & 1 & 0.872 \\
0.01 & 3.808 & 3.653 & 2.919 & 1 & 0.884 \\
0.1 & 1.845 & 1.803 & 1.584 & 1 & 0.954 \\
0.5 & 1.106 & 1.088 & 1.074 & 1 & 0.959 \\
1 & 1.056 & 1.033 & 1.041 & 1 & 0.960 \\
2 & 1.042 & 1.005 & 1.044 & 1 & 0.956
\end{tabular}

DGP: BFF

\begin{tabular}{llllll}
0 & 4.162 & 3.990 & 3.376 & 1.527 & 1 \\
0.01 & 4.006 & 3.832 & 3.185 & 1.483 & 1 \\
0.1 & 1.861 & 1.801 & 1.627 & 1.145 & 1 \\
0.5 & 1.143 & 1.120 & 1.110 & 1.051 & 1 \\
1 & 1.110 & 1.078 & 1.102 & 1.062 & 1 \\
2 & 1.088 & 1.053 & 1.088 & 1.048 & 1 \\
\hline
\end{tabular}


Table 4: Estimation results for a threecountry BFF model, standard errors in parentheses.

\begin{tabular}{|c|c|c|c|c|}
\hline & & USA & CAN & JPN \\
\hline & & Diffus & n charact & ristics \\
\hline & $p$ & 0.0366 & 0.0389 & 0.0935 \\
\hline & & $(0.0195)$ & $(0.0172)$ & $(0.0335)$ \\
\hline & $q$ & 0.3004 & 0.3916 & 0.5141 \\
\hline & & $(0.0887)$ & $(0.0862)$ & $(0.1016)$ \\
\hline & $m$ & 0.9048 & 0.8537 & 0.9411 \\
\hline & & $(0.1235)$ & $(0.0707)$ & $(0.0117)$ \\
\hline & & & depends & \\
\hline & & USA & CAN & JPN \\
\hline & USA & 0.156 & 0.326 & 0.135 \\
\hline 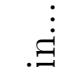 & & $(0.253)$ & $(0.217)$ & (0.107) \\
\hline శี & CAN & -1.068 & 1.254 & -0.036 \\
\hline $\overrightarrow{\underline{n}}$ & & $(0.37)$ & $(0.268)$ & $(0.160)$ \\
\hline$\ddot{\circ}$ & JPN & -0.479 & 0.048 & 1.002 \\
\hline & & $(0.216)$ & $(0.128)$ & $(0.356)$ \\
\hline
\end{tabular}




\section{References}

Bass, F. M. (1969), A New Product Growth Model for Consumer Durables, Management Science, 15, 215-227.

Bass, F. M., T. V. Krishnan, and D. C. Jain (1994), Why the Bass Model Fits without Decision Variables, Marketing Science, 13, 203-223.

Boswijk, H. P. and P. H. Franses (2005), On the Econometrics of the Bass Diffusion Model, Journal of Business \& Economic Statistics, 23, 255-268.

Kumar, V. and T. V. Krishnan (2002), Multinational Diffusion Models: An Alternative Framework, Marketing Science, 21, 318-330.

Putsis, W. P., Jr., S. Balasubramanian, E. H. Kaplan, and S. K. Sen (1997), Mixing Behavior in Cross-Country Diffusion, Marketing Science, 16, 354-369. 


\section{Tinbergen Institute}

The Tinbergen Institute is the institute for economic research of the Erasmus Universiteit Rotterdam, Universiteit van Amsterdam, and Vrije Universiteit Amsterdam.

Tinbergen Institute Amsterdam

Roetersstraat 31

1018 WB Amsterdam

The Netherlands

Tel.: $\quad+31(0) 205513500$

Fax: $\quad+31(0) 205513555$

Tinbergen Institute Rotterdam

Burg. Oudlaan 50

3062 PA Rotterdam

The Netherlands

Tel.: $\quad+31(0) 104088900$

Fax: $\quad+31(0) 104089031$

Please send questions and/or remarks of nonscientific nature to driessen@tinbergen.nl.

Most TI discussion papers can be downloaded at http://www.tinbergen.nl. 